\title{
Stochastic Control-Oriented Modeling of Flexible Air-Breathing Hypersonic Vehicle
}

\author{
Yan-rui Wu $\left(\mathbb{D},{ }^{1}\right.$ Peng-fei Yang ${ }^{(D},{ }^{2}$ and You-li Wu ${ }^{1}{ }^{1}$ \\ ${ }^{1}$ School of Aeronautics Engineering, Air Force Engineering University, Xian 710038, China \\ ${ }^{2}$ Research Center for Evaluation and Argumentation, Academy of Military Science, Beijing 100091, China \\ Correspondence should be addressed to Peng-fei Yang; pfyang1988@126.com
}

Received 6 December 2017; Revised 26 March 2018; Accepted 3 April 2018; Published 8 May 2018

Academic Editor: Luis J. Yebra

Copyright (c) 2018 Yan-rui Wu et al. This is an open access article distributed under the Creative Commons Attribution License, which permits unrestricted use, distribution, and reproduction in any medium, provided the original work is properly cited.

\begin{abstract}
The flexible dynamics of commonly used air-breathing hypersonic vehicle model are not tractable for control design and the inevitable stochastic perturbations are usually neglected. Aiming at these deficiencies, reduced flexible dynamics are deducted in this paper and a stochastic control-oriented vehicle model is established accordingly. The responses of the original system to the deterministic and the stochastic part of the generalized force, which is treated as the input of the flexible dynamic system, are analyzed. After that, the simplified flexible dynamics is deduced to approximate the responses. The reduced flexible dynamics, which are tractable for control design since they greatly reduce the complexity of the original dynamics, are comprised of a simple function of the determined generalized force and an Ornstein-Uhlenbeck colored noise. Finally, the longitudinal dynamics in parametric strict feedback form are obtained by substituting the reduced flexible dynamics into the original model. The applicability of the simplified flexible dynamics is validated through the numerical simulations.
\end{abstract}

\section{Introduction}

Air-breathing hypersonic vehicle (AHV) is a reliable and cost-effective technology for access to space and providing rapid global response capability; thus considerable efforts have been made to the further development and design of AHV [1-4]. Notwithstanding the success of NASA's X-43A and X-51A experimental vehicle and the applying of advanced control strategies [5-8], the design of guidance and control systems for hypersonic vehicles is still an open problem $[9$, 10].

The modeling of the AHV dynamics is a challenging task due to various reasons [11, 12]. First, the strong coupling between propulsive and aerodynamic forces results from the location of the scramjet engine. Second, the flexible effects are caused by the length and slender geometry. In addition, significant uncertainties and stochastic noises affect the vehicle, as a result of the variability of its characteristics with the flight conditions, fuel consumption, thermal effects, and measurement noises of sensors [11, 13, 14].

Many efforts have been made to develop simulation models for AHV to match the real-world physics. The main results focus on the longitudinal dynamics, since the AHV rarely takes transverse maneuvering limited by the scramjet engine $[4,13,14]$. The first attempt to build an analytical AHV model was made by Chavez and Schmidt [15]. After that, Clark et al. employed computational fluid dynamics (CFD) and analytical analysis to model the longitudinal dynamics [16]. Then a nonlinear, physics-based model of the longitudinal dynamics for AHV called the first-principle model (FPM) was proposed by Bolender and Doman, which captures a number of complex interactions between the propulsion system, aerodynamics, and structural dynamics [11]. To overcome the analytical intractability of FPM, a simplified control-oriented model (COM) was obtained by Parker et al. [17]. Considering that the flexible effects are coupled into the pitch rate equation in FPM and COM, Williams et al. modified the flexible dynamics and got the decoupled COM (DCOM) using the assumed-modes method [18]. Both the COM and DCOM have been widely used in the controller design [19-23] since it retains the relevant dynamical features of the FPM and offers the advantage of being analytically tractable. The subsequent dynamic models of AHV are mostly deduced from COM and DCOM $[24,25]$. 


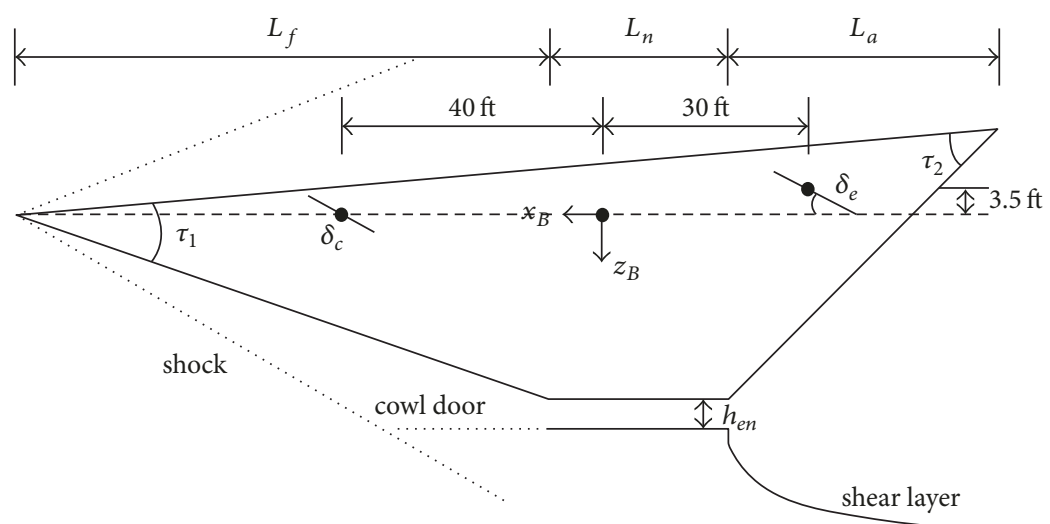

FIGURE 1: The side view of X-43A.

However, DCOM is still quite complex for controller design since the flexible dynamic equations are second-order systems, which is hard to substitute into the rigid dynamic and there are no well-developed methods to directly get the controller. Hence the existing controller developed based on DCOM makes use of feedback from the rigid states only and keeps the flexible states "frozen" at nominal trim condition, meaning that the flexible dynamics are ignored during the controller design $[23,26,27]$. In recent years, Krylov subspace methods have been employed to compute reduced-order models of high-order linear time invariant systems [28, 29], which have been used for reduced-order modeling of large-scale dynamical systems [30]. The reducedorder modeling for the flexible dynamics of AHV has not been researched. Another significant drawback is that none of the existing AHV models considers the inevitable stochastic perturbations caused by the airflow, operational error of aerodynamic control surfaces, and measurement noise of sensors.

By analyzing the above-mentioned deficiencies, this paper modified the longitudinal dynamics of DCOM proposed in [18] and simplified the flexible dynamics. Meanwhile, the stochastic perturbations are taken into consideration during the simplification in order to establish a stochastic decoupled control-oriented AHV model (SDCOM). The reduced flexible dynamics greatly reduce the complexity of the original dynamics. The proposed stochastic AHV model is derived from physics-based model and it is tractable for stochastic control design. The rest of this paper is organized as follows: the longitudinal dynamics of DCOM are briefly introduced in Section 2. The responses of flexible dynamics to deterministic signal and Gaussian white noise signal are analyzed. Then the flexible dynamics are simplified in Section 3. The longitudinal dynamics of SDCOM are established in Section 4. The numerical simulations are given in Section 5 to illustrate the validity of the simplified system.

\section{The Decoupled Control-Oriented Model}

A sketch of the AHV geometry is shown in Figure 1 and the detailed structure parameters can be seen in $[11,17,18]$.

The DCOM was developed by Williams et al. [18] on the bases of FPM proposed by Bolender and Doman [11]; the longitudinal dynamics are derived using Lagrange's equations and compressible flow theory. The assumed-modes method [31] is employed to establish the flexible dynamic equations. Unlike COM, the flexible modes are orthogonal to the rigid body modes; therefore the interaction between rigid and flexible dynamics occurs only through the aerodynamic forces. The scramjet engine model is adopted from Chavez and Schmidt [15]. The longitudinal dynamics of DCOM written in the stability axis coordinate system are

$$
\begin{aligned}
& \dot{V}=\frac{T \cos \alpha-D}{m}-g \sin \gamma \\
& \dot{h}=V \sin \gamma \\
& \dot{\gamma}=\frac{L+T \sin \alpha}{m V}-\frac{g}{V} \cos \gamma \\
& \dot{\alpha}=-\frac{L+T \sin \alpha}{m V}+Q+\frac{g}{V} \cos \gamma \\
& \dot{Q}=\frac{M}{I_{y y}} \\
& \ddot{\eta}_{i}=-2 \zeta_{i} \omega_{i} \dot{\eta}_{i}-\omega_{i}^{2} \eta_{i}+N_{i}, \quad i=1,2,3,
\end{aligned}
$$

where $T, D, L, M$ are thrust, drag, lift, and pitching moment, respectively; $V, h, \gamma, \alpha, Q$ represent the vehicle velocity, flight height, flight-path angle, angle of attack, and pitch rate, respectively; $\eta_{i}$ denotes the $i$ th flexible generalized coordinate, which is the proportion of the $i$ th flexible mode shape; $\zeta_{i}$ represents the $i$ th structure damping ratio; $\omega_{i}$ is the $i$ th structure natural frequency; $N_{i}$ is the $i$ th generalized force. The complicated computation of the aerodynamic forces are replaced with curve-fitted approximations as follows $[18,26$, 32]:

$$
\begin{aligned}
T & \approx \bar{q} S\left[C_{T, \phi}(\alpha) \phi+C_{T}(\alpha)+C_{T}^{\eta} \boldsymbol{\eta}\right] \\
L & \approx \bar{q} S C_{L}(\alpha, \boldsymbol{\delta}, \boldsymbol{\eta}) \\
D & \approx \bar{q} S C_{D}(\alpha, \boldsymbol{\delta}, \boldsymbol{\eta}) \\
M & \approx z_{T} T+\bar{q} \bar{c} S C_{M}(\alpha, \boldsymbol{\delta}, \boldsymbol{\eta}) \\
N_{i} & \approx \bar{q} S C_{N_{i}}(\alpha, \boldsymbol{\delta}, \boldsymbol{\eta}), \quad i=1,2,3,
\end{aligned}
$$


where $\bar{q}=0.5 \rho V^{2}$ is the dynamic pressure and $\rho$ is the air density; $z_{T}$ is the thrust-to-moment coupling coefficient; $\bar{c}$ is the mean aerodynamic chord; $S$ denotes the reference area; $\boldsymbol{\delta}=\left[\delta_{e}, \delta_{c}\right]^{T}$ represents elevator angular deflection and canard angular deflection; $\phi$ represents the fuel equivalence ratio. The coefficients are

$$
\begin{aligned}
& C_{T, \phi}(\alpha)= C_{T}^{\phi \alpha^{3}} \alpha^{3}+C_{T}^{\phi \alpha^{2}} \alpha^{2}+C_{T}^{\phi \alpha} \alpha+C_{T}^{\phi} \\
& C_{T}(\alpha)= C_{T}^{\alpha^{3}} \alpha^{3}+C_{T}^{\alpha^{2}} \alpha^{2}+C_{T}^{\alpha} \alpha+C_{T}^{0} \\
& C_{L}(\alpha, \boldsymbol{\delta}, \boldsymbol{\eta})= C_{L}^{\alpha} \alpha+C_{L}^{\delta_{e}} \delta_{e}+C_{L}^{\delta_{c}} \delta_{c}+C_{L}^{0}+C_{L}^{\eta} \boldsymbol{\eta} \\
& C_{D}(\alpha, \boldsymbol{\delta}, \boldsymbol{\eta})= C_{D}^{\alpha^{2}} \alpha^{2}+C_{D}^{\alpha} \alpha+C_{D}^{\delta_{e}^{2}} \delta_{e}^{2}+C_{D}^{\delta_{e}} \delta_{e}+C_{D}^{\delta_{c}^{2}} \delta_{c}^{2} \\
&+C_{D}^{\delta_{c}} \delta_{c}+C_{D}^{0}+C_{D}^{\eta} \boldsymbol{\eta} \\
& C_{M}(\alpha, \boldsymbol{\delta}, \boldsymbol{\eta})= C_{M}^{\alpha^{2}} \alpha^{2}+C_{M}^{\alpha} \alpha+C_{M}^{\delta_{e}} \delta_{e}+C_{M}^{\delta_{c}} \delta_{c}+C_{M}^{0} \\
&+C_{M}^{\eta} \boldsymbol{\eta} \\
& C_{N_{i}}= N_{i}^{\alpha^{2}} \alpha^{2}+N_{i}^{\alpha} \alpha+N_{i}^{\delta_{e}} \delta_{e}+N_{i}^{\delta_{c}} \delta_{c}+N_{i}^{0}, \\
& i=1,2,3 .
\end{aligned}
$$

The parameters and coefficients can be found in [32]. It is worth noting that all of the coefficients of the DCOM are subject to uncertainty. The role of the canard deflection is to adaptively decouple lift from elevator commands, so the following relationship holds to eliminate the nonminimum phase behavior [33]:

$$
\delta_{c}=-\frac{C_{L}^{\delta_{e}}}{C_{L}^{\delta_{c}}} \delta_{e} .
$$

\section{The Simplification of Flexible Dynamics}

It can be seen from the DCOM model given in Section 2 that the flexible dynamic equations are

$$
\begin{aligned}
& \ddot{\eta}_{i}+2 \zeta_{i} \omega_{i} \dot{\eta}_{i}+\omega_{i}^{2} \eta_{i}=N_{i} \\
& N_{i} \approx \bar{q} S\left[N_{i}^{\alpha^{2}} \alpha^{2}+N_{i}^{\alpha} \alpha+N_{i}^{\delta_{e}} \delta_{e}+N_{i}^{\delta_{c}} \delta_{c}+N_{i}^{0}\right] .
\end{aligned}
$$

It is obvious that the flexible dynamics are second-order linear systems and the flexible states $\eta_{i}$ are the responses of the system to the input signal. Notice that the value of $N_{i}$ is inevitably affected by stochastic perturbations caused by the airflow, operational errors, and measurement noises, we separate $N_{i}$ into two kinds of signals: the deterministic signal $N_{i}^{d}$ and the stochastic signal $N_{i}^{n}$. Then the responses of the flexible dynamics to $N_{i}$ can be accordingly separated into two parts: $\eta_{i}^{d}$ and $\eta_{i}^{n}$, which represent the responses to $N_{i}^{d}$ and $N_{i}^{n}$, respectively. Since the first three flexible states $\eta_{i}$ have the similar expression and decoupled with each other, hereafter we take $\eta_{1}$, for example, to explain the simplification process.
3.1. Response to Deterministic Signal. In this section, the input signal of system (5) is considered as the deterministic steady signal $N_{i}^{d}$. Let $\mathbf{x}=\left[\begin{array}{ll}\eta_{1}^{d} & \dot{\eta}_{1}^{d}\end{array}\right]^{T}$ and (5) can be rewritten as

$$
\begin{aligned}
& \dot{x}_{1}=x_{2} \\
& \dot{x}_{2}=N_{1}^{d}-2 \zeta_{1} \omega_{1} x_{2}-\omega_{1}^{2} x_{1} .
\end{aligned}
$$

The above equation can be expressed as

$$
\begin{gathered}
\dot{\mathbf{x}}=\mathbf{A x}+\mathbf{B} u, \\
\mathbf{x}\left(t_{0}\right)=\left[\begin{array}{ll}
\eta_{10}^{d} & \dot{\eta}_{10}^{d}
\end{array}\right]^{T},
\end{gathered}
$$

where

$$
\begin{aligned}
& \mathbf{A}=\left[\begin{array}{cc}
0 & 1 \\
-\omega_{1}^{2} & -2 \zeta_{1} \omega_{1}
\end{array}\right], \\
& \mathbf{B}=\left[\begin{array}{l}
0 \\
1
\end{array}\right], \\
& u=N_{1}^{d} .
\end{aligned}
$$
$e^{\mathbf{A} t}:$

We can use inverse Laplace transform tables to compute

$$
e^{\mathbf{A} t}=\mathscr{L}^{-1}\left[(s \mathbf{I}-\mathbf{A})^{-1}\right],
$$

where

$$
\begin{aligned}
(s \mathbf{I}-\mathbf{A})^{-1} & =\left[\begin{array}{cc}
s & -1 \\
\omega_{1}^{2} & s+2 \zeta_{1} \omega_{1}
\end{array}\right]^{-1} \\
& =\left[\begin{array}{cc}
\frac{s+2 \zeta_{1} \omega_{1}}{s^{2}+2 \zeta_{1} \omega_{1} s+\omega_{1}^{2}} & \frac{1}{s^{2}+2 \zeta_{1} \omega_{1} s+\omega_{1}^{2}} \\
\frac{-\omega_{1}^{2}}{s^{2}+2 \zeta_{1} \omega_{1} s+\omega_{1}^{2}} & \frac{s}{s^{2}+2 \zeta_{1} \omega_{1} s+\omega_{1}^{2}}
\end{array}\right] .
\end{aligned}
$$

Define the following notations:

$$
\begin{aligned}
& E_{11} \\
& =\left[\cosh \left(\omega_{1} \sqrt{\zeta_{1}^{2}-1} t\right)+\frac{\zeta_{1} \sinh \left(\omega_{1} \sqrt{\zeta_{1}^{2}-1} t\right)}{\sqrt{\zeta_{1}^{2}-1}}\right] \\
& E_{12}=\frac{\sin \left(\omega_{1} \sqrt{1-\zeta_{1}^{2}} t\right)}{\omega_{1} \sqrt{1-\zeta_{1}^{2}}}
\end{aligned}
$$




$$
\begin{aligned}
& E_{21}=-\frac{\omega_{1} \sin \left(\omega_{1} \sqrt{1-\zeta_{1}^{2}} t\right)}{\sqrt{1-\zeta_{1}^{2}}} \\
& E_{22} \\
& =\left[\cosh \left(\omega_{1} \sqrt{\zeta_{1}^{2}-1 t}\right)-\frac{\zeta_{1} \sinh \left(\omega_{1} \sqrt{\zeta_{1}^{2}-1} t\right)}{\sqrt{\zeta_{1}^{2}-1}}\right]
\end{aligned}
$$

and we have

$$
e^{\mathbf{A} t}=e^{-\zeta_{1} \omega_{1} t}\left[\begin{array}{ll}
E_{11} & E_{12} \\
E_{21} & E_{22}
\end{array}\right] .
$$

Substitute (14) into (10) to obtain the analytical solution of $\eta_{1}^{d}$ :

$$
\begin{aligned}
\eta_{1}^{d} & =\frac{N_{1}^{d}}{\omega_{1}^{2}}+e^{-\zeta_{1} \omega_{1} t}\left\{\eta_{10} E_{11}+\left(\dot{\eta}_{10}^{d}+\frac{N_{1}^{d} \zeta_{1}}{\omega_{1}}\right) E_{12}\right. \\
& \left.-\frac{N_{1}^{d}}{\omega_{1}^{2}} \cos \left(\omega_{1} \sqrt{1-\zeta_{1}^{2} t}\right)\right\} .
\end{aligned}
$$

From (15), we can conclude that $\eta_{1}^{d}$ converges exponentially to $N_{1}^{d} / \omega_{1}^{2}$; the rate of convergence is determined by $\zeta_{1} \omega_{1}$. It is worth noting that the results above are got under the assumption that $N_{1}^{d}$ is steady, which is surely not accurate. However, from the results got by Oppenheimer et al. [35] and Bolender and Doman [11], the natural frequencies of the rigid body states range from $0.09 \mathrm{rad} / \mathrm{s}$ to $4 \mathrm{rad} / \mathrm{s}$, which are far less than the natural frequencies of the flexible states, which range from $15 \mathrm{rad} / \mathrm{s}$ to $120 \mathrm{rad} / \mathrm{s}$ [18]. So the main factors on $N_{1}^{d}$ change far slower than the rate of convergence of $\eta_{1}^{d}$. From the engineering practical view, it is reasonable to assume that $N_{1}^{d}$ is steady within a small time interval.

3.2. Response to Stochastic Signal. In the practical flight process, the stochastic perturbations caused by the turbulent flow and measurement errors of sensors are modeled as zeromean Gaussian white noise [36]. Assume that the stochastic part of $i$ th generalized force $N_{i}^{n}$ is a zero-mean Gaussian white noise $\xi_{i}(t)$ and $\xi_{i}(t) \sim N\left(0, \sigma_{i}^{2} \delta(t)\right)$, where $\delta(t)$ is the Dirac's delta function. Denote the standard Gaussian white noise as $\xi(t) \sim N(0, \delta(t))$; then we have

$$
\xi_{i}(t)=\left[\xi_{1}(t), \xi_{2}(t), \xi_{3}(t)\right]^{T}=\left[\sigma_{1}, \sigma_{2}, \sigma_{3}\right]^{T} \xi(t) .
$$

Assume that the response of flexible dynamic system to the stochastic input $\xi_{1}(t)$ is $\eta_{1}^{n}$, so the following equation holds:

$$
\ddot{\eta}_{1}^{n}+2 \zeta_{1} \omega_{1} \dot{\eta}_{1}^{n}+\omega_{1}^{2} \eta_{1}^{n}=\sigma_{1} \xi(t)
$$

According to stochastic process theory [37], $\eta_{1}^{n}$ is a colored noise because the Gaussian white noise is the input of the second-order dynamic system. This colored noise is not tractable for stochastic control design, so it is necessary to simplify the system. In practical application, the OrnsteinUhlenbeck colored noise, which is a response of a first-order dynamic system to Gaussian white noise, is commonly used and has received a great amount of research [38, 39]. The ultimate purpose of this section is to construct an OrnsteinUhlenbeck process, which has distribution characteristics similar to $\eta_{1}^{n}$.

Let $a>0$ and $b=$ const.; the following Itô stochastic differential equation

$$
\mathrm{d} \chi_{1}=-a \chi_{1} \mathrm{~d} t+b \mathrm{~d} w(t), \quad \chi_{1}\left(t_{0}\right)=0
$$

is called the Langevin equation, where $w(t)$ is 1-dimensional standard Brownian motion. The solution stochastic process $\chi_{1}(t)(t \geq 0)$ is called the Ornstein-Uhlenbeck process, which is a colored noise. It is obvious that the Ornstein-Uhlenbeck process is the response of a first-order system to Gaussian white noise signal.

Let the input noise signal $\xi(t)$ be a constant $c$ to explore the solution of systems (17) and (18); the analytical solution of (17) can be got as the following according to the solving process in Section 3.1:

$$
\begin{aligned}
\eta_{1}^{n} & =\frac{\sigma_{1} c}{\omega_{1}^{2}}+e^{-\zeta_{1} \omega_{1} t}\left\{\eta_{10}^{n} E_{11}+\left(\dot{\eta}_{10}^{n}+\frac{\sigma_{1} c \zeta_{1}}{\omega_{1}}\right) E_{12}\right. \\
& \left.-\frac{\sigma_{1} c}{\omega_{1}^{2}} \cos \left(\omega_{1} \sqrt{1-\zeta_{1}^{2}} t\right)\right\},
\end{aligned}
$$

where $\eta_{10}^{n}, \dot{\eta}_{10}^{n}$ are initial values of $\eta_{1}^{n}$ and $\dot{\eta}_{1}^{n} ; E_{11}, E_{12}$ are given in (13).

Then consider the following differential equation:

$$
\dot{\chi}_{1}+a \chi_{1}=b c, \quad \chi_{10}=0
$$

It is easy to give the analytical solution of (20) as follows [40]:

$$
\chi_{1}(t)=\frac{b c}{a}-e^{-a t} \frac{b c}{a} .
$$

The task is to find proper parameters $a$ and $b$ to achieve that the stochastic processes $\chi_{1}(t)$ and $\eta_{1}^{n}$ have the similar distribution. By comparing (19) and (21), the following equations can be deduced to guarantee the same convergent rate and the convergent steady value:

$$
\begin{aligned}
a & =\zeta_{1} \omega_{1} \\
\frac{b c}{a} & =\frac{\sigma_{1} c}{\omega_{1}^{2}} .
\end{aligned}
$$

So we have

$$
\begin{aligned}
& a=\zeta_{1} \omega_{1} \\
& b=\frac{\zeta_{1} \sigma_{1}}{\omega_{1}} .
\end{aligned}
$$


From the above points, we can conclude that the solution process $\chi_{1}(t)$ of the following Langevin equation

$$
\mathrm{d} \chi_{1}=-\zeta_{1} \omega_{1} \mathrm{~d} t+\frac{\zeta_{1} \sigma_{1}}{\omega_{1}} \mathrm{~d} w(t), \quad \chi_{1}\left(t_{0}\right)=0
$$

has the distribution characteristics similar to the stochastic process $\eta_{1}^{n}$.

Synthesize the results in Sections 3.1 and 3.2; the reduced flexible generalized coordinates $\eta_{i}$ in the longitudinal dynamics (1) can be obtained as

$$
\widehat{\eta}_{i}(t)=\eta_{i}^{d}(t)+\eta_{i}^{n}(t) \approx \frac{N_{i}^{d}}{\omega_{i}^{2}}+\chi_{i}, \quad i=1,2,3
$$

where $\chi_{i}$ are determined by the following differential equations:

$$
\mathrm{d} \chi_{1}=-\zeta_{1} \omega_{1} \mathrm{~d} t+\frac{\zeta_{1} \sigma_{1}}{\omega_{1}} \mathrm{~d} w(t), \quad \chi_{1}\left(t_{0}\right)=0
$$

Expression (25), which greatly reduces the complexity of the former flexible states dynamics, is comprised of a simple function of the determined generalized force and an Ornstein-Uhlenbeck colored noise. It is tractable for control design since there are many well-developed methods to handle the Ornstein-Uhlenbeck process [37].

\section{The Longitudinal Dynamics of Stochastic AHV Model}

Considering that some flight parameters of AHV can also suffer from stochastic perturbations, the stochastic perturbations of $\gamma, \alpha, Q$ are modeled as zero-mean Gaussian white noises, which are denoted as $w_{\gamma}, w_{\alpha}, w_{\mathrm{Q}}$, respectively [36]. The high-order terms and the cross-terms are omitted for convenience. Substitute expression (25) into the AHV longitudinal dynamics (1) to get the following nonlinear system in parametric strict feedback form:

$$
\begin{aligned}
& \dot{V}=f_{V}+g_{V} \phi+\Delta f_{V}+\mathbf{h}_{V} \chi+H_{V} w_{\alpha} \\
& \dot{h}=V \sin \gamma+V w_{\gamma} \\
& \dot{\gamma}=f_{\gamma}+g_{\gamma} \alpha+\Delta f_{\gamma}+\mathbf{h}_{\gamma} \chi+H_{\gamma} w_{\alpha} \\
& \dot{\alpha}=f_{\alpha}+g_{\alpha} Q+\Delta f_{\alpha}+\mathbf{h}_{\alpha} \boldsymbol{\chi}+H_{\alpha} w_{\alpha}+w_{\mathrm{Q}} \\
& \dot{Q}=f_{\mathrm{Q}}+g_{\mathrm{Q}} \delta_{e}+\Delta f_{\mathrm{Q}}+\mathbf{h}_{\mathrm{Q}} \chi+H_{\mathrm{Q}} w_{\alpha} \\
& \dot{\chi}_{i}=-\zeta_{i} \omega_{i} \chi_{i}+\frac{\zeta_{i} \sigma_{i}}{\omega_{i}} \xi(t), \quad i=1,2,3,
\end{aligned}
$$

where $\Delta f_{*}(*=V, \gamma, \alpha, Q)$ represents the uncertainties including parameter perturbations of the curve-fitted coefficients, the high-order terms and cross-terms omitted during the deduction, and so on; $\boldsymbol{\chi}=\left[\chi_{1}, \chi_{2}, \chi_{3}\right]^{T}$; other functions and symbols in (27) are introduced as follows.

(1) The Velocity $V$ Equation

$$
\begin{aligned}
f_{V} & =\frac{\bar{q} S}{m}\left[\cos \alpha\left(C_{T}^{\alpha} \alpha+C_{T}^{0}\right)-C_{D}^{\alpha} \alpha\right. \\
& \left.-\left(C_{D}^{\delta_{e}}-\frac{C_{D}^{\delta_{c}} C_{L}^{\delta_{e}}}{C_{L}^{\delta_{c}}}\right) \delta_{e}-C_{D}^{0}\right]-g \sin \gamma+f_{V}^{\eta} \\
g_{V} & =\frac{\bar{q} S}{m} \cos \alpha\left(C_{T}^{\phi \alpha} \alpha+C_{T}^{\phi}\right), \\
\mathbf{h}_{V}= & \frac{\bar{q} S}{m}\left[h_{V}^{1}, h_{V}^{2}, h_{V}^{3}\right], \\
H_{V}= & \frac{\bar{q} S}{m}\left\{\left[\cos \alpha C_{T}^{\alpha}+C_{T}^{\phi \alpha} \phi\right]-C_{D}^{\alpha}\right\},
\end{aligned}
$$

where

$$
\begin{aligned}
& f_{V}^{\eta}=\frac{\bar{q} S}{m} \sum_{i=1}^{3}\left[\left(\cos \alpha C_{T}^{\eta_{i}}-C_{D}^{\eta_{i}}\right) \frac{N_{i}}{\omega_{i}^{2}}\right], \\
& h_{V}^{i}=\cos \alpha C_{T}^{\eta_{i}}-C_{D}^{\eta_{i}},
\end{aligned}
$$

$$
i=1,2,3 .
$$

(2) The Flight-Path Angle $\gamma$ Equation

$$
\begin{aligned}
& f_{\gamma}=\frac{\bar{q} S}{m V}\left[C_{L}^{0}+\sin \alpha\left(C_{T}^{\phi} \phi+C_{T}^{0}\right)\right]-\frac{g}{V} \cos \gamma+f_{\gamma}^{\eta} \\
& g_{\gamma}=\frac{\bar{q} S}{m V}\left(C_{L}^{\alpha}+C_{T}^{\phi \alpha} \phi+C_{T}^{\alpha}\right), \\
& \mathbf{h}_{\gamma}=\frac{\bar{q} S}{m V}\left[h_{\gamma}^{1}, h_{\gamma}^{2}, h_{\gamma}^{3}\right] \\
& H_{\gamma}=\frac{\bar{q} S}{m V}\left\{C_{L}^{\alpha}+\sin \alpha \phi C_{T}^{\phi \alpha}+\sin \alpha C_{T}^{\alpha}\right\},
\end{aligned}
$$

where

$$
\begin{aligned}
& f_{\gamma}^{\eta}=\frac{\bar{q} S}{m V} \sum_{i=1}^{3}\left[\left(\sin \alpha C_{T}^{\eta_{i}}+C_{L}^{\eta_{i}}\right) \frac{N_{i}}{\omega_{i}^{2}}\right], \\
& h_{\gamma}^{i}=\sin \alpha C_{T}^{\eta_{i}}+C_{L}^{\eta_{i}},
\end{aligned}
$$

$$
i=1,2,3 \text {. }
$$

(3) The Angle of Attack $\alpha$ Equation

$$
\begin{aligned}
f_{\alpha} & =-\frac{\bar{q} S}{m V}\left[C_{L}^{0}+C_{L}^{\alpha} \alpha+C_{T}^{\phi \alpha} \phi \alpha+C_{T}^{\alpha} \alpha\right. \\
& \left.+\sin \alpha\left(C_{T}^{\phi} \phi+C_{T}^{0}\right)\right]+\frac{g}{V} \cos \gamma+f_{\alpha}^{\eta} \\
g_{\alpha} & =1, \\
\mathbf{h}_{\alpha} & =\frac{\bar{q} S}{m V}\left[h_{\alpha}^{1}, h_{\alpha}^{2}, h_{\alpha}^{3}\right], \\
H_{\alpha} & =-\frac{\bar{q} S}{m V}\left[C_{L}^{\alpha}+\sin \alpha C_{T}^{\phi \alpha} \phi+\sin \alpha C_{T}^{\alpha}\right],
\end{aligned}
$$


TABLE 1: The maximum value of $\left|e_{i}^{m}(t)\right|$ and $\left|e_{i}^{D}(t)\right|$.

\begin{tabular}{ccccccc}
\hline & $\left|e_{1}^{m}(t)\right|$ & $\mid e_{1}^{D}(t)$ & $\left|e_{2}^{m}(t)\right|$ & $\left|e_{2}^{D}(t)\right|$ & $\left|e_{3}^{m}(t)\right|$ & $\left|e_{3}^{D}(t)\right|$ \\
\hline Maximum value & 0.016 & 0.03 & 0.0041 & 0.0062 & 0.0013 & 0.0028 \\
\hline
\end{tabular}

where

$$
\begin{aligned}
& f_{\alpha}^{\eta}=\frac{\bar{q} S}{m V} \sum_{i=1}^{3}\left[\left(C_{L}^{\eta_{i}}-\sin \alpha C_{T}^{\eta_{i}}\right) \frac{N_{i}}{\omega_{i}^{2}}\right], \\
& h_{\alpha}^{i}=C_{L}^{\eta_{i}}-\sin \alpha C_{T}^{\eta_{i}},
\end{aligned}
$$

$$
i=1,2,3 \text {. }
$$

(4) The Pitch Rate $Q$ Equation

$$
\begin{aligned}
f_{\mathrm{Q}} & =\frac{\bar{q} S}{I_{y y}}\left[z_{T}\left(C_{T}^{\phi \alpha} \phi \alpha+C_{T}^{\phi} \phi+C_{T}^{\alpha} \alpha+C_{T}^{0}\right)\right. \\
& \left.+\bar{c}\left(C_{M}^{\alpha^{2}} \alpha^{2}+C_{M}^{\alpha} \alpha+C_{M}^{0}\right)\right]+f_{\mathrm{Q}}^{\eta} \\
g_{\mathrm{Q}} & =\frac{\bar{q} S \bar{c}}{I_{y y}}\left(C_{M}^{\delta_{e}}-\frac{C_{M}^{\delta_{c}} C_{L}^{\delta_{e}}}{C_{L}^{\delta_{c}}}\right), \\
\mathbf{h}_{\mathrm{Q}} & =\frac{\bar{q} S}{m V}\left[h_{\mathrm{Q}}^{1}, h_{\mathrm{Q}}^{2}, h_{\mathrm{Q}}^{3}\right], \\
H_{\mathrm{Q}} & =\frac{\bar{q} \bar{c} S}{I_{y y}}\left\{C_{M}^{\alpha}\right\},
\end{aligned}
$$

where

$$
\begin{aligned}
& f_{\mathrm{Q}}^{\eta}=\frac{\bar{q} S}{I_{y y}} \sum_{i=1}^{3}\left[\left(z_{T} C_{T}^{\eta_{i}}+\bar{c} C_{M}^{\eta_{i}}\right) \frac{N_{i}}{\omega_{i}^{2}}\right], \\
& h_{\mathrm{Q}}^{i}=z_{T} C_{T}^{\eta_{i}}+\bar{c} C_{M}^{\eta_{i}},
\end{aligned}
$$

$$
i=1,2,3 \text {. }
$$

It can be seen from the above equations that expression (27) is not in the strict feedback form since the term $\sin \alpha$ is included in the function $f_{\gamma}$, and the control input $\delta_{e}$ enters functions $f_{\gamma}, f_{\mathrm{Q}}$, and $f_{\alpha}$ through the term $N_{i}$. However, there are some reasons why we can still allege that the system (27) is suitable for backstepping control. First, the natural frequencies of the $\gamma$-equation is larger than $\alpha$-equation, so that the term $\sin \alpha$ can be treated as constant. Second, the aerodynamic control surfaces have significant inertial delay [41], meaning that $\delta_{e}$ can be seen as constant when calculating the generalized force $N_{i}$. Third, it is assumed that all model parameters are subject to uncertainty up to $40 \%$, and the necessary significations are considered acceptable in order to design guidance and control systems for AHV [23].

To sum up, the proposed stochastic AHV model possesses many advantages: first, the control design is more convenient since the strong coupling between the flexible states and the rigid body states and the control variables are canceled; second, the distribution of the colored noise is known and the system is in parametric strict feedback form, so the control can be easily designed based on a combination of backstepping and stochastic system theory.

\section{Numerical Simulations}

The reduced form (25) of the flexible generalized coordinate $\eta_{i}$ has been tested in simulation to validate the correctness of our results obtained in the previous section. The first three structure natural frequencies and damping ratios are set as the following according to literatures $[26,35]$ :

$$
\begin{aligned}
\boldsymbol{\omega} & =[19.7437,47.7851,94.8203]^{T} \mathrm{rad} / \mathrm{s} \\
\zeta & =[0.7,0.6,0.5]^{T} .
\end{aligned}
$$

5.1. Response to Gaussian White Noise. The stochastic noise intensity affecting the generalized force $N_{i}$ is significant since the dynamic pressure of AHV during the flight is very large. For simplicity, we take the Gaussian white noise inputs as $\xi_{i}(t) \sim N\left(0,100^{2} \delta(t)\right)$ to compare the responses of the original second-order flexible dynamics (17) and the simplified first-order dynamics (24).

Notice that $\eta_{i}^{n}(t)$ and $\chi_{i}(t)$ are both stochastic processes, which is a collection of random variables, so the characteristics of them must have been investigated from two aspects: one is the time characteristics of $\eta_{i}^{n}(t)$ and $\chi_{i}(t)$; another one is the distribution properties of $\eta_{i}^{n}(t)$ and $\chi_{i}(t)$ at fixed time $t_{\text {fix }}$.

For the time characteristics, we investigate the numerical characteristic functions of $\eta_{i}^{n}(t)$ and $\chi_{i}(t)$. The expectations of $\eta_{i}^{n}(t)$ and $\chi_{i}(t)$ are denoted as $m_{i}^{\eta}(t)$ and $m_{i}^{\chi}(t)$, respectively; The standard deviations of $\eta_{i}^{n}(t)$ and $\chi_{i}(t)$ are denoted as $D_{i}^{\eta}(t)$ and $D_{i}^{\chi}(t)$, respectively. Then 1000 Monte Carlo runs have been done to obtain $m_{i}^{\eta}(t), m_{i}^{\chi}(t), D_{i}^{\eta}(t), D_{i}^{\chi}(t)$. The error curves $e_{i}^{m}(t)=m_{i}^{\eta}(t)-m_{i}^{\chi}(t)$ and $e_{i}^{D}(t)=D_{i}^{\eta}(t)-D_{i}^{\chi}(t)$ are shown in Figure 2 to compare the characteristics. The maximum values of $\left|e_{i}^{m}(t)\right|$ and $\left|e_{i}^{D}(t)\right|$ are listed in Table 1 . From these results, we can see that numerical characteristics of the stochastic process $\eta_{i}^{n}(t)$ and $\chi_{i}(t)$ are very close to each other; the reduced system has similar response to the Gaussian white noise to the original system.

As for the distribution properties of $\eta_{i}^{n}(t)$ and $\chi_{i}(t)$ at fixed time, 10000 Monte Carlo runs have been done and the deviations between the statistical distributions of $\eta_{i}^{n}(t)$ and $\chi_{i}(t)$ at time $t_{\text {fix }}=10 \mathrm{~s}$ are given in Figure 3.

We can see that the statistical results of $\chi_{i}\left(t_{\text {fix }}\right)$ at a given time are very close to $\eta_{i}^{n}\left(t_{\text {fix }}\right)$. The statistical distribution comparison between $\eta_{i}^{n}\left(t_{\text {fix }}\right)$ and $\chi_{i}\left(t_{\text {fix }}\right)$ is given in Figure 4 to illustrate the distinctness.

The results show that the statistical distributions of $\chi_{i}(t)$ at a given time are similar to $\eta_{i}^{n}(t)$, especially the first-order flexible generalized coordinate $\eta_{1}^{n}(t)$, which is the main factor 


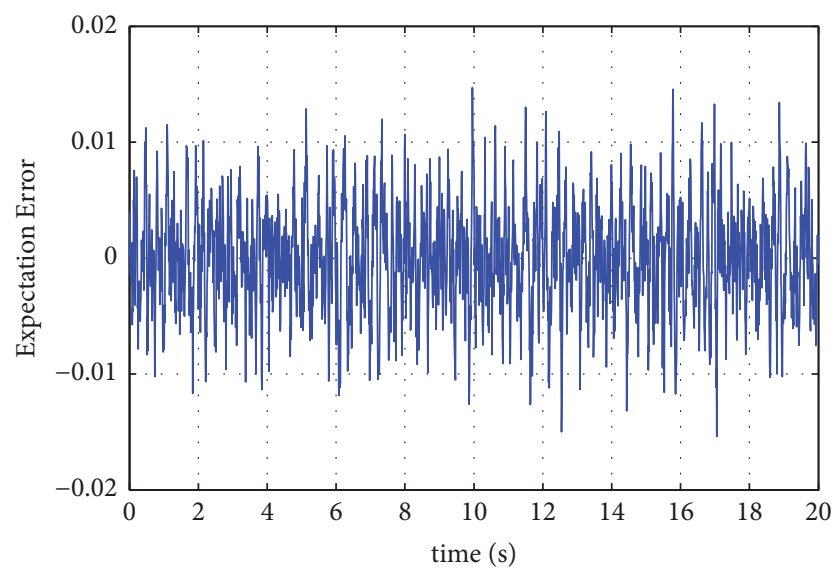

(a) $e_{1}^{m}(t)$

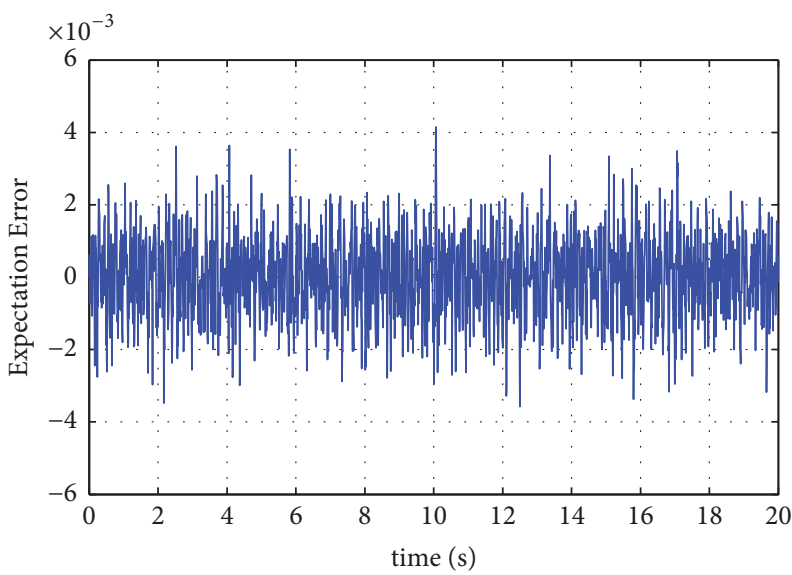

(c) $e_{2}^{m}(t)$

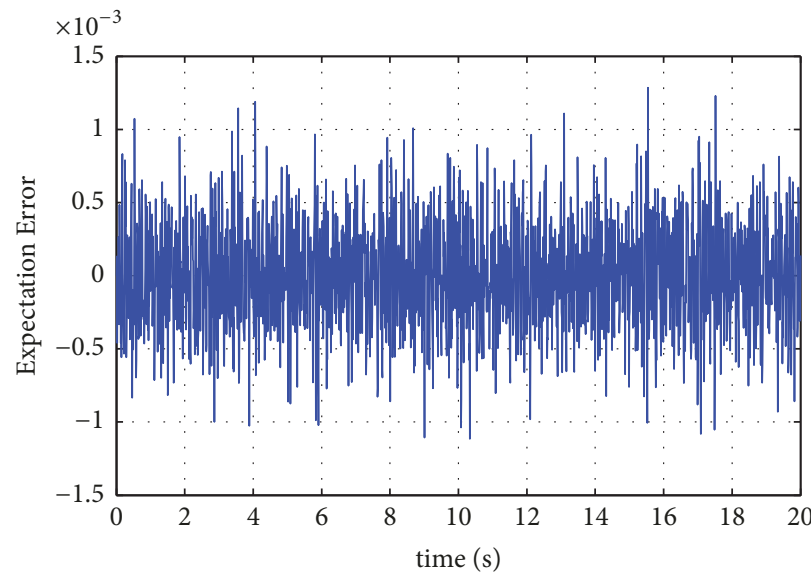

(e) $e_{3}^{m}(t)$

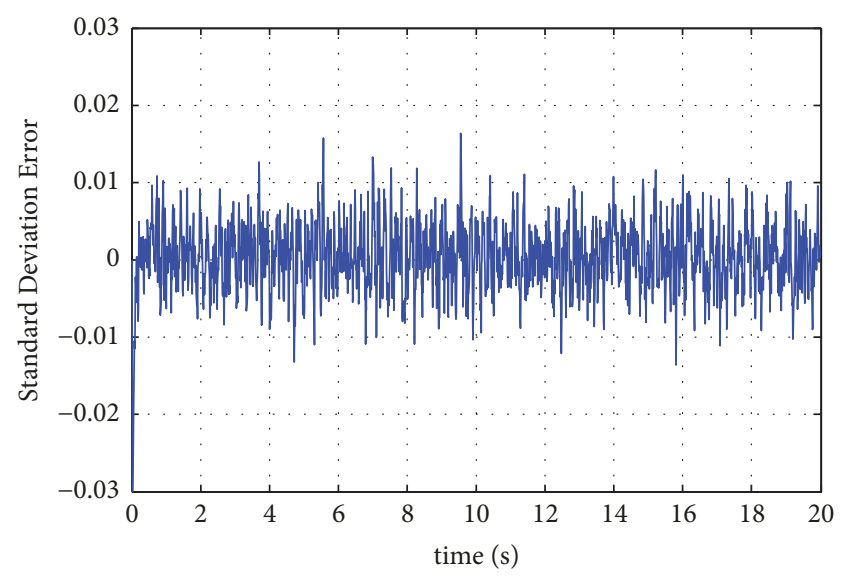

(b) $e_{1}^{D}(t)$

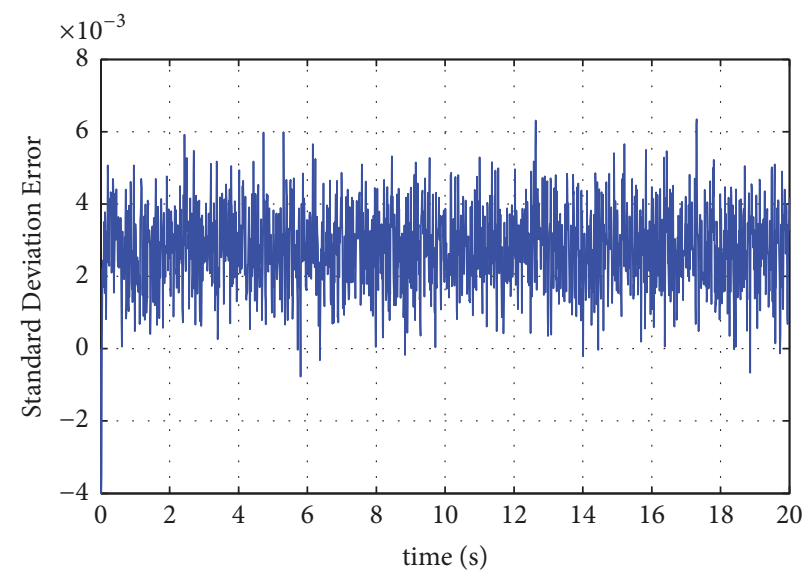

(d) $e_{2}^{D}(t)$

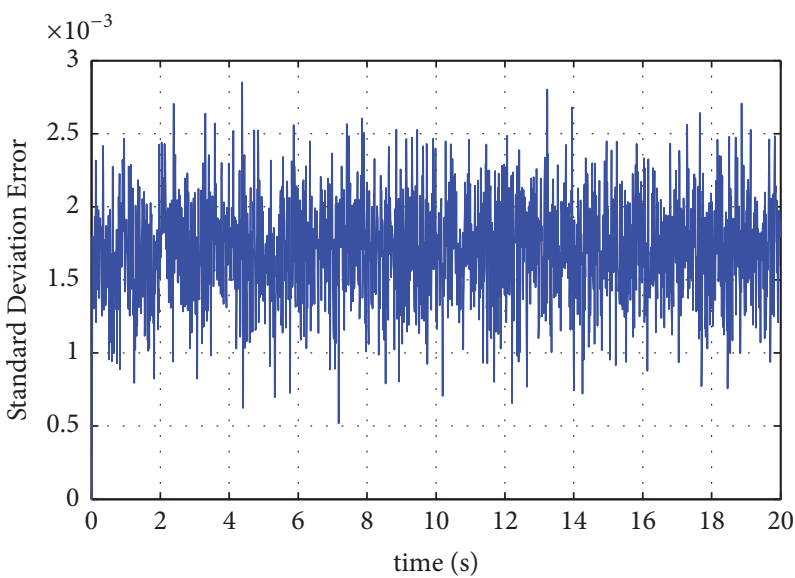

(f) $e_{3}^{D}(t)$

Figure 2: The expectation and standard deviation error of $\eta_{i}^{n}(t)$ and $\chi_{i}(t)$.

of the flexible states. From the above results, we can draw the conclusion that the reduced system (24) can be used to replace the flexible dynamics (17) for the Gaussian white noise inputs.

5.2. Response to Stochastic Time-Varying Signal Input. The previous section only validates the response to the stochastic parts of the generalized force $N_{i}$. As discussed before, the generalized force $N_{i}$ is comprised of two parts: the deterministic part $N_{i}^{d}$ and the stochastic part $N_{i}^{n}$. In this section, the input signal is assumed to be a stochastic time-varying signal to meet the requirements of the reality. The generalized force $N_{i}$ is chosen as the following based on the admissible ranges of the states and parameters. 


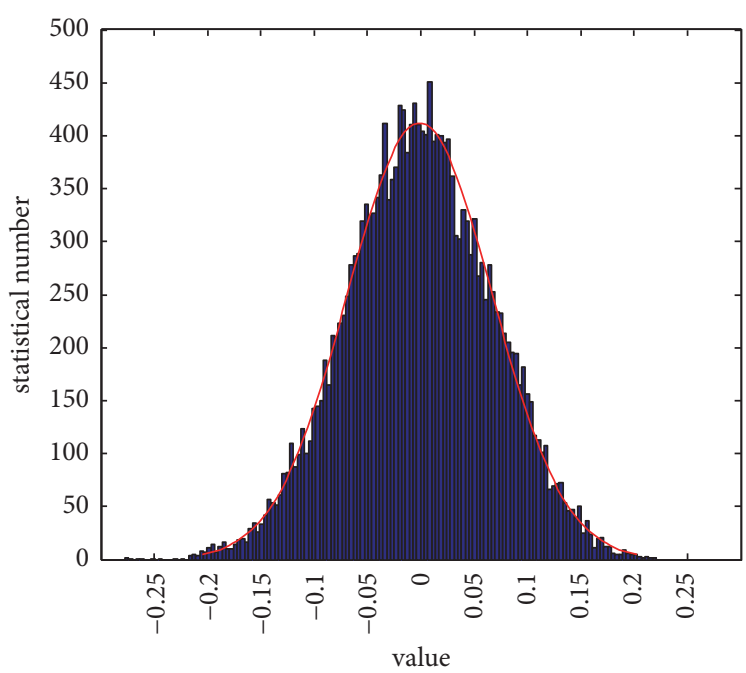

(a) $\eta_{1}^{n}\left(t_{\text {fix }}\right)$

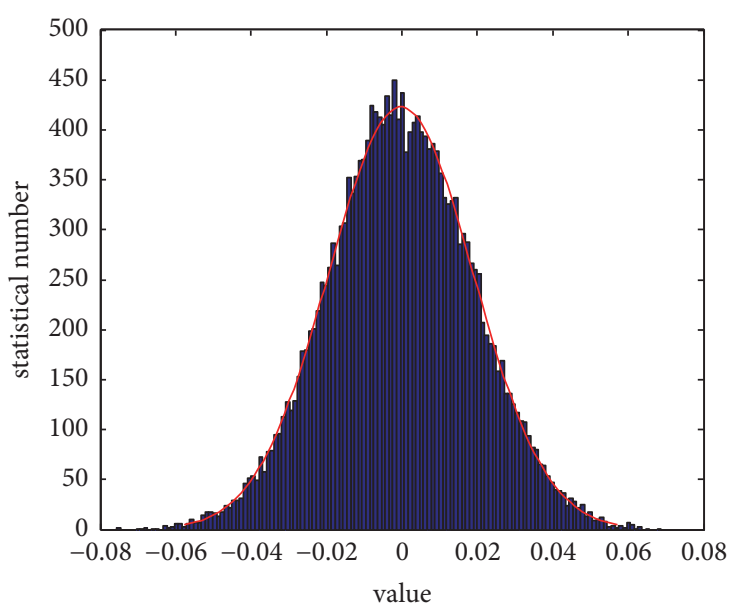

(c) $\eta_{2}^{n}\left(t_{\text {fix }}\right)$

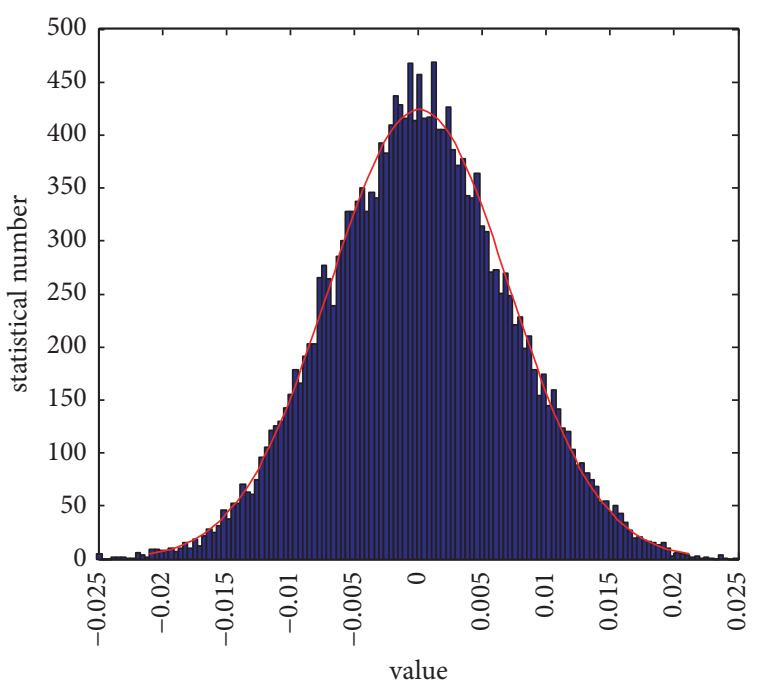

(e) $\eta_{3}^{n}\left(t_{\text {fix }}\right)$

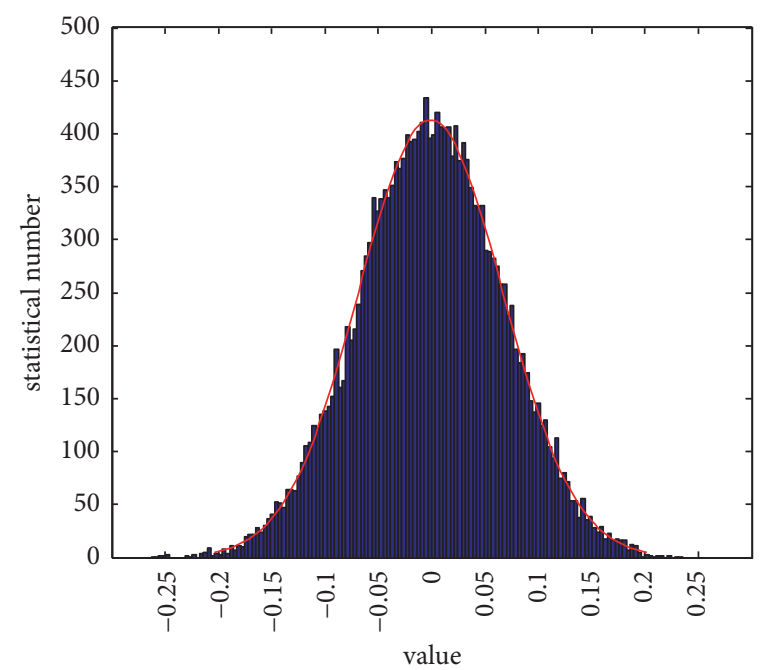

(b) $\chi_{1}\left(t_{\text {fix }}\right)$

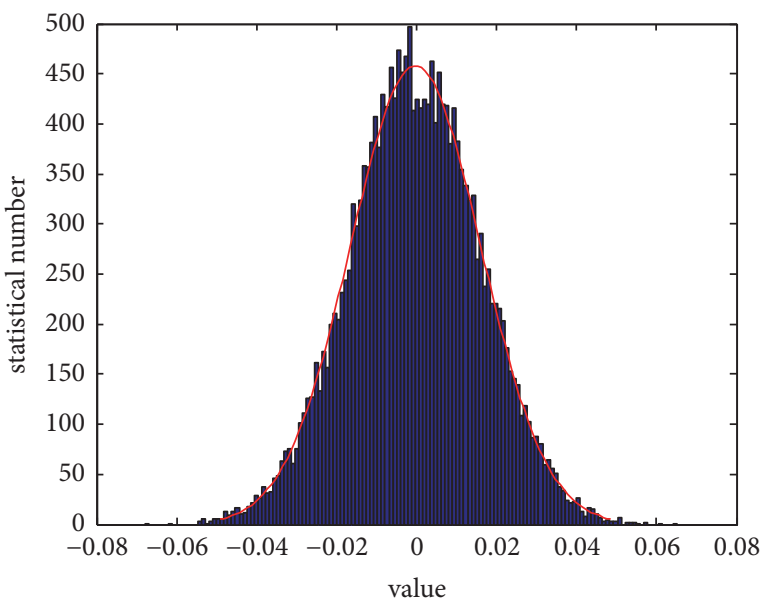

(d) $\chi_{2}\left(t_{\text {fix }}\right)$

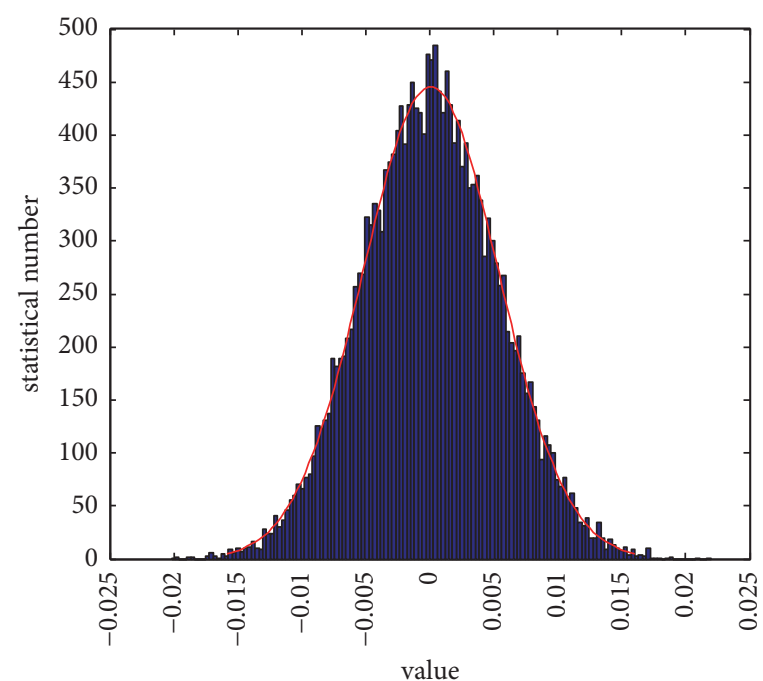

(f) $\chi_{3}\left(t_{\text {fix }}\right)$

FIGURE 3: Statistical results of $\eta_{i}^{n}\left(t_{\text {fix }}\right)$ and $\chi_{i}\left(t_{\text {fix }}\right), t_{\text {fix }}=10 \mathrm{~s}$. 


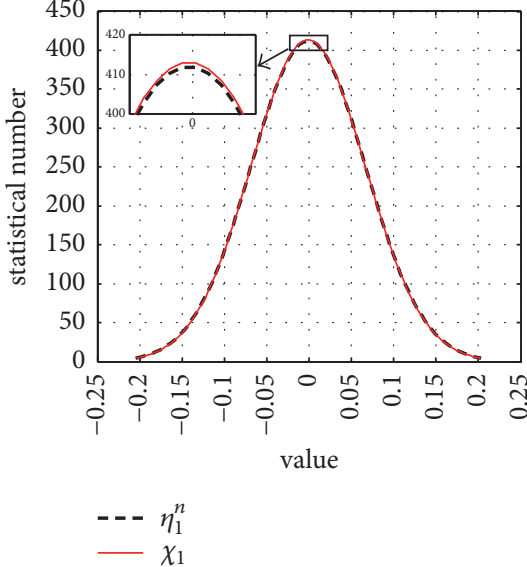

(a) $\eta_{1}^{n}\left(t_{\text {fix }}\right)$ and $\chi_{1}\left(t_{\text {fix }}\right)$

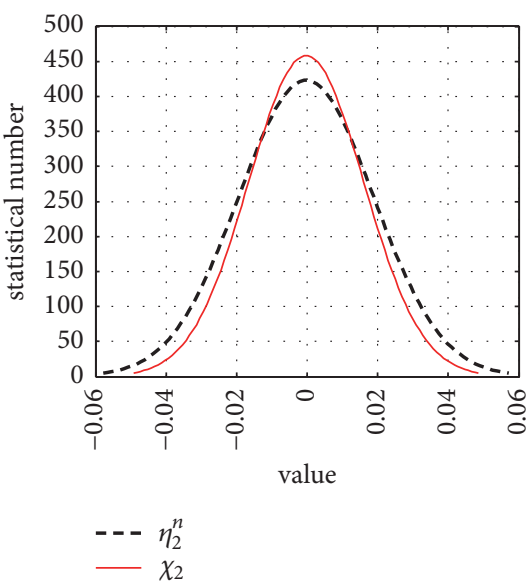

(b) $\eta_{2}^{n}\left(t_{\text {fix }}\right)$ and $\chi_{2}\left(t_{\text {fix }}\right)$

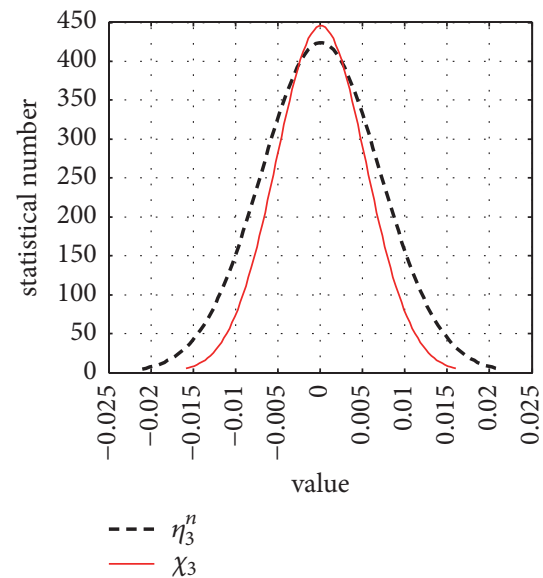

(c) $\eta_{3}^{n}\left(t_{\text {fix }}\right)$ and $\chi_{3}\left(t_{\text {fix }}\right)$

FIGURE 4: Statistical distribution comparison between $\eta_{i}^{n}\left(t_{\mathrm{fix}}\right)$ and $\chi_{i}\left(t_{\mathrm{fix}}\right) t_{\mathrm{fix}}=10 \mathrm{~s}$.
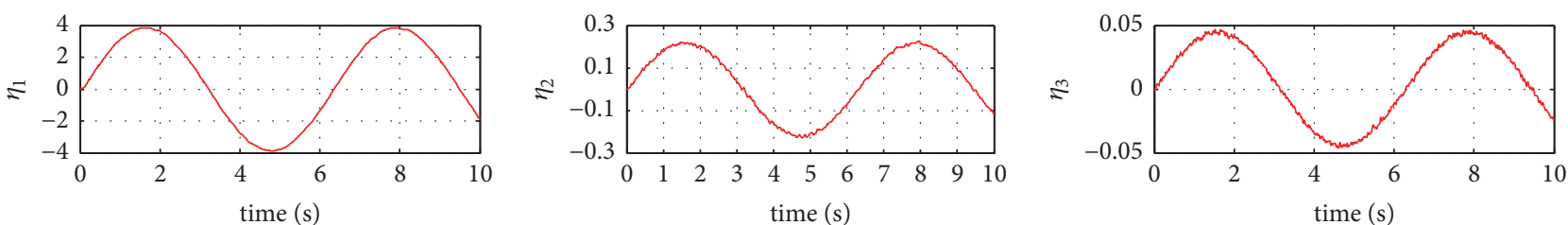

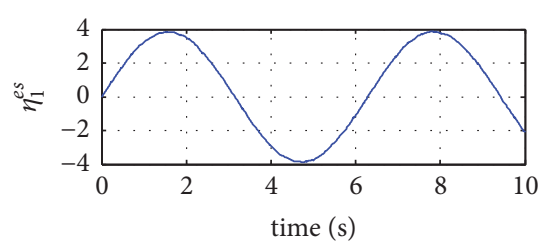

(a) $\eta_{1}(t)$ and $\widehat{\eta}_{1}(t)$

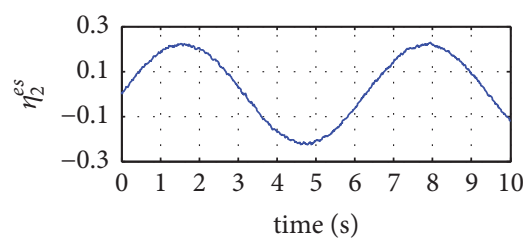

(b) $\eta_{2}(t)$ and $\widehat{\eta}_{2}(t)$

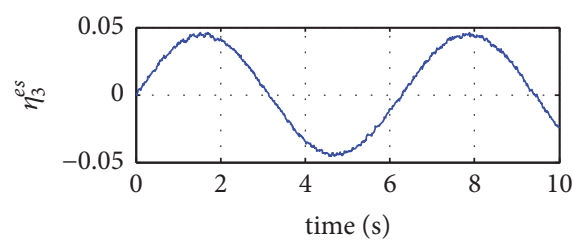

(c) $\eta_{3}(t)$ and $\hat{\eta}_{3}(t)$

FIGURE 5: Comparison between $\eta_{i}(t)$ and $\widehat{\eta}_{i}(t)$.

$$
\begin{aligned}
& N_{1}(t)=1500 \sin (t)+\xi_{1}(t) \\
& N_{2}(t)=500 \sin (t)+\xi_{2}(t) \\
& N_{3}(t)=400 \sin (t)+\xi_{3}(t),
\end{aligned}
$$

where $\xi_{1}(t) \sim N\left(0,17^{2} \delta(t)\right), \xi_{2}(t) \sim N\left(0,10^{2} \delta(t)\right), \xi_{3}(t) \sim$ $N\left(0,10^{2} \delta(t)\right)$. The original output $\eta_{i}(t)$ and its approximation $\widehat{\eta}_{i}(t)$ are calculated according to (5) and (25), respectively. The results are plotted in Figure 5.

From Figure 5 we can see that the reduced flexible system responses are basically the same as the original ones to the stochastic time-varying input signal. However, the original flexible states need to be calculated by a far more complicated flexible dynamics system. The applicability of the theoretical development has been illustrated through the simulations above.

5.3. Analysis of the Longitudinal Dynamics. In this section, the behavior of open-loop longitudinal dynamics of SDCOM (27) is analyzed using geometric control technique. The models taken for comparison are FPM developed by Bolender and
Doman [11], COM developed by Parker et al. [17], and DCOM proposed by Williams et al. [18].

The output of the models was selected as $y=[V, \gamma]^{T}$ and the control inputs are $u=\left[\delta_{e}, \phi\right]^{T}$. First, the longitudinal dynamics were trimmed at $M a=8$ and $h=85,000 \mathrm{ft}$. The trim condition is given in Table 2 , where $\dot{\eta}_{i}$ and $\dot{\eta}_{i}$ are the flexible states of DCOM and SDCOM; $\eta_{f}, \eta_{a}, \dot{\eta}_{f}, \dot{\eta}_{a}$ represent the flexible states of FPM and COM.

The dynamics of AHV models have been linearized around the trim condition and the poles and zeros of the linearized systems are shown in Figure 6.

The actual pole and zero locations of the abovementioned models are given in Table 3.

Figures 6(a) and 6(b) show that both FPM and COM have 7 zeros and 9 poles and the locations of zeros and poles are very close. The two complex conjugate pairs correspond to the flexible dynamics, indicating that they are minimum phase. The pair of zeros that appears to be symmetric about the imaginary axis correspond to the $\gamma$ dynamics and one of them is nonminimum phase. The zero at the origin corresponds to the altitude state. In view of Figures 6(c) and 6(d), it is obvious that DCOM and SDCOM have 9 zeros and 11 poles. The three 


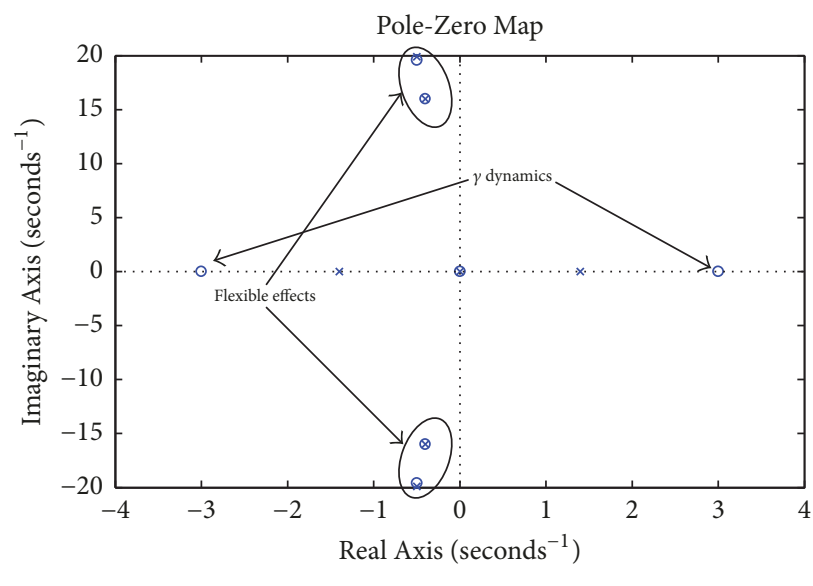

$\times$ Poles

- Zeros

(a) FPM

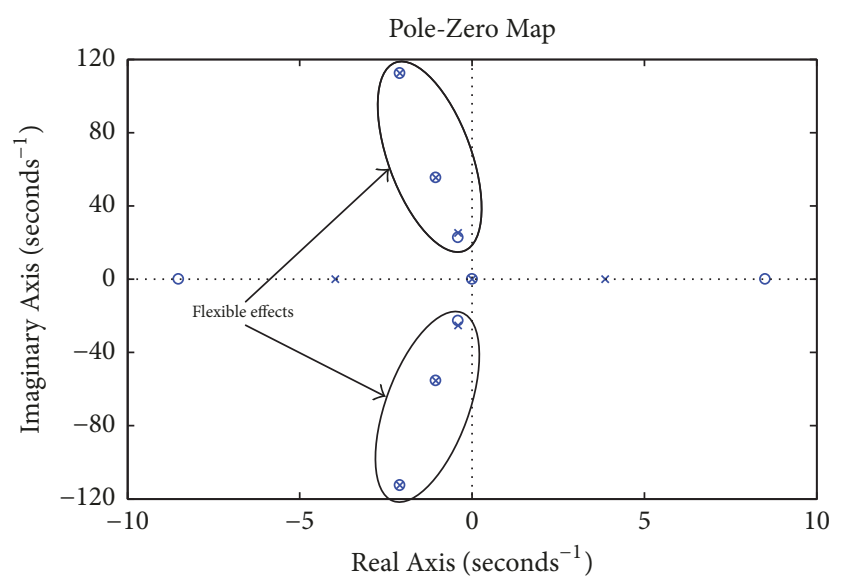

$\times$ Poles

$\circ$ Zeros

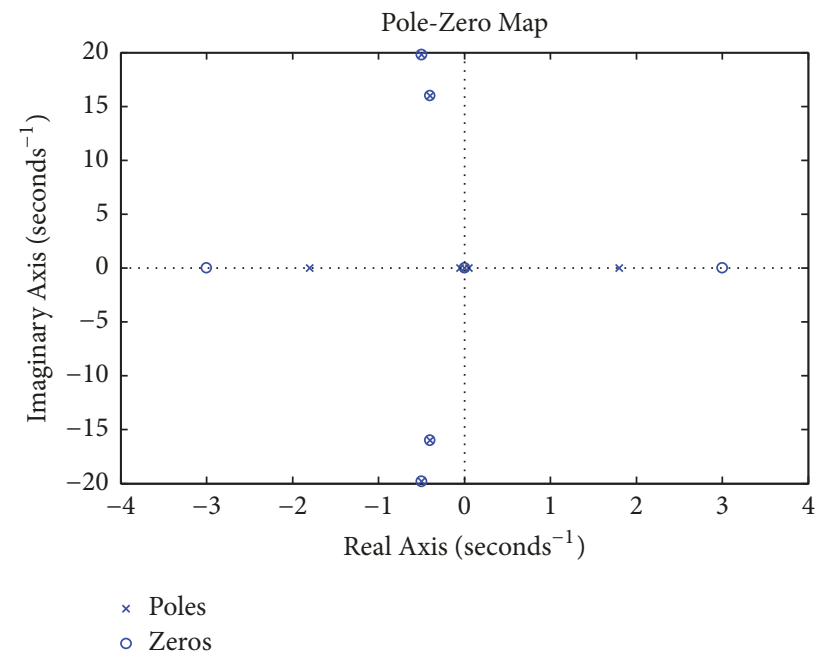

(b) $\mathrm{COM}$

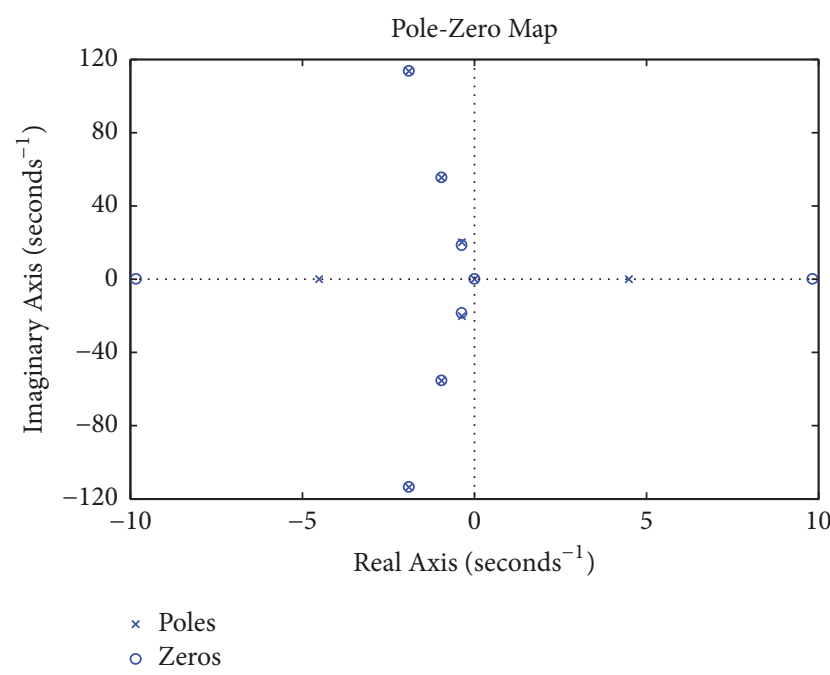

(d) SDCOM

(c) DCOM

Figure 6: Pole-zero map comparison.

TABLE 2: Trim condition for the AHV model.

\begin{tabular}{|c|c|c|c|c|c|}
\hline State & Value & State & Value & Input & Value \\
\hline V & $85,000 \mathrm{ft}$ & $\dot{\eta}_{2}$ & 0 & $\phi$ & 0.2514 \\
\hline$h$ & $7702.08 \mathrm{ft} \cdot \mathrm{s}^{-1}$ & $\eta_{3}\left(\chi_{3}\right)$ & $0.029 \mathrm{ft}$ & $\delta_{e}$ & 11.4635 \\
\hline$\gamma$ & 0 deg & $\dot{\eta}_{3}$ & 0 & & \\
\hline$\alpha$ & $1.5153 \mathrm{deg}$ & & & & \\
\hline Q & $0 \mathrm{deg} \cdot \mathrm{s}^{-1}$ & $\eta_{f}$ & $1.5122 \mathrm{ft}$ & & \\
\hline$\eta_{1}\left(\chi_{1}\right)$ & $0.827 \mathrm{ft}$ & $\dot{\eta}_{f}$ & 0 & & \\
\hline$\dot{\eta}_{1}$ & 0 & $\eta_{a}$ & $1.2114 \mathrm{ft}$ & & \\
\hline$\eta_{2}\left(\chi_{2}\right)$ & $0.185 \mathrm{ft}$ & $\dot{\eta}_{a}$ & 0 & & \\
\hline
\end{tabular}

complex conjugate pairs correspond to the flexible dynamics, indicating that they are minimum phase. The poles and zeros of rigid body dynamics move away from the imaginary axis compared to the FPM and COM, but the dynamical characteristics change only a little. So we can draw the conclusion that COM, DCOM, and SDCOM approximate the behavior of the FPM; the open-loop longitudinal dynamics of AHV are unstable and possess nonminimum phase characteristic.

\section{Conclusions}

Reduced flexible dynamics of the existing flexible AHV model have been deducted in this paper and a stochastic 
TABLE 3: Poles and zeros of the models.

\begin{tabular}{|c|c|c|c|c|c|c|c|}
\hline \multicolumn{2}{|l|}{ TM } & \multicolumn{2}{|c|}{$\mathrm{COM}$} & \multicolumn{2}{|c|}{ DCOM } & \multicolumn{2}{|c|}{ SDCOM } \\
\hline Poles & Zeros & Poles & Zeros & Poles & Zeros & Poles & Zeros \\
\hline$-0.54 \pm 19.9 i$ & $-0.54 \pm 19.6 i$ & $-0.54 \pm 19.6 i$ & $-0.54 \pm 19.6 i$ & $-2.1 \pm 112.5 i$ & $-2.1 \pm 112.5 i$ & $-1.9 \pm 113.5 i$ & $-1.9 \pm 113.5 i$ \\
\hline$-0.35 \pm 16.2 i$ & $-0.36 \pm 16.2 i$ & $-0.35 \pm 16.2 i$ & $-0.36 \pm 16.2 i$ & $-1.05 \pm 55.4 i$ & $-1.05 \pm 55.4 i$ & $-0.95 \pm 55.4 i$ & $-0.95 \pm 55.4 i$ \\
\hline-1.41 & 2.98 & -1.83 & 2.98 & $-0.41 \pm 25.2 i$ & $-0.41 \pm 22.8 i$ & $-0.36 \pm 20.1 i$ & $-0.36 \pm 18.5 i$ \\
\hline 1.41 & -2.98 & 1.83 & -2.98 & -3.97 & -8.52 & -4.51 & -9.83 \\
\hline-0.00042 & 0 & -0.00058 & 0 & 3.86 & 8.52 & 4.49 & 9.83 \\
\hline$-7.5 \times 10^{-5} \pm 0.03 i$ & & $-0.05 \pm 0.02 i$ & & $\begin{array}{c}0.00056 \\
6.2 \times 10^{-4} \pm 0.001\end{array}$ & 0 & $\begin{array}{c}0.00037 \\
5.1 \times 10^{-4} \pm 0.00\end{array}$ & 0 \\
\hline
\end{tabular}

control-oriented AHV model has been established. The flexible dynamic system of the traditional DCOM has been analyzed firstly by decomposing the generalized force into two parts. The simplified flexible dynamics have been deduced to approximate the responses to each part of the generalized force. Finally, the longitudinal dynamics of stochastic AHV model in parametric strict feedback form have been obtained by substituting the reduced form flexible dynamics into the original DCOM model. The applicability of the simplified flexible dynamics has been validated through numerical simulations.

However, there still exist some limits of the proposed model: the coefficients of the model are subject to uncertainty up to $40 \%$, which should be eliminated by real-word experiments; in the proposed model, only the stochastic elements in the flexible dynamics are considered, which can be improved by adding stochastic noises into the rigid body dynamics so that the model would be more realistic; another important work is to develop real-world examples.

\section{Conflicts of Interest}

The authors declare that they have no conflicts of interest.

\section{Acknowledgments}

This work has been supported by the Major Program of the National Natural Science Foundation of China (no. 61627901).

\section{References}

[1] C. Mu, Z. Ni, C. Sun, and H. He, "Air-breathing hypersonic vehicle tracking control based on adaptive dynamic programming," IEEE Transactions on Neural Networks and Learning Systems, vol. 28, no. 3, pp. 584-598, 2017.

[2] X. Bu, X. Wu, J. Huang, Z. Ma, and R. Zhang, "Minimallearning-parameter based simplified adaptive neural backstepping control of flexible air-breathing hypersonic vehicles without virtual controllers," Neurocomputing, vol. 175, pp. 816$825,2016$.

[3] R. Aditya, M. J. Balas, and D. B. Doman, "Direct adaptive stability \& command augmentation of an air-breathing hypersonic vehicle," in Proceedings of the IEEE Aerospace Conference, pp. 112, Big Sky, Mont, USA, March 2016.
[4] D. Preller, M. K. Smart, and D. Geller, "Longitudinal control strategy for hypersonic accelerating vehicles," Journal of Spacecraft and Rockets, vol. 52, no. 3, pp. 993-998, 2015.

[5] T. Wang, J. Qiu, S. Yin, H. Gao, J. Fan, and T. Chai, "Performance-based adaptive fuzzy tracking control for networked industrial processes," IEEE Transactions on Cybernetics, vol. 46, no. 8, pp. 1760-1770, 2016.

[6] J. Qiu, H. Gao, and S. X. Ding, "Recent advances on fuzzymodel-based nonlinear networked control systems: a survey," IEEE Transactions on Industrial Electronics, vol. 63, no. 2, pp. 1207-1217, 2016.

[7] T. Wang, J. Qiu, and H. Gao, "Adaptive neural control of stochastic nonlinear time-delay systems with multiple constraints," IEEE Transactions on Systems, Man, and Cybernetics: Systems, vol. 47, no. 8, pp. 1875-1883, 2017.

[8] T. Wang, H. Gao, and J. Qiu, "A combined fault-tolerant and predictive control for network-based industrial processes," IEEE Transactions on Industrial Electronics, vol. 63, no. 4, pp. 2529-2536, 2016.

[9] A. Serrani and M. A. Bolender, "Addressing limits of operability of the scramjet engine in adaptive control of a generic hypersonic vehicle," in Proceedings of the 55th IEEE Conference on Decision and Control, pp. 7567-7572, Las Vegas, Nev, USA, December 2016.

[10] P. Papadopoulos, E. Venkatapathy, D. Prabhu, M. P. Loomis, and D. Olynick, "Current grid-generation strategies and future requirements in hypersonic vehicle design, analysis and testing," Applied Mathematical Modelling, vol. 23, no. 9, pp. 705-735, 1999.

[11] M. A. Bolender and D. B. Doman, "Nonlinear longitudinal dynamical model of an air-breathing hypersonic vehicle," Journal of Spacecraft and Rockets, vol. 44, no. 2, pp. 374-387, 2007.

[12] F. Piscitelli, L. Cutrone, G. Pezzella, P. Roncioni, and M. Marini, "Nose-to-tail analysis of an airbreathing hypersonic vehicle using an in-house simplified tool," Acta Astronautica, vol. 136, pp. 148-158, 2017.

[13] M. A. Bolender, "An overview on dynamics and controls modelling of hypersonic vehicles," in Proceedings of the American Control Conference (ACC '09), pp. 2507-2512, IEEE, St. Louis, Mo, USA, June 2009.

[14] B.-J. Tsai and Y.-T. Chou, "Analyzing the longitudinal effect of hypersonic flow past a conical cone via the perturbation method," Applied Mathematical Modelling, vol. 32, no. 12, pp. 2596-2620, 2008.

[15] F. R. Chavez and D. K. Schmidt, "Analytical aeropropulsive/aeroelastic hypersonic-vehicle model with dynamic analysis," Journal of Guidance, Control, and Dynamics, vol. 17, no. 6, pp. 1308-1319, 1994. 
[16] A. D. Clark, M. D. Mirmirani, C. Wu, S. Choi, and M. Kuipers, "An aero-propulsion integrated elastic model of a generic airbreathing hypersonic vehicle," in Proceedings of the AIAA Guidance, Navigation, and Control Conference and Exhibit, pp. 3699-3718, Keystone, Colo, USA, August 2006.

[17] J. T. Parker, A. Serrani, S. Yurkovich, M. A. Bolender, and D. B. Doman, "Control-oriented modeling of an air-breathing hypersonic vehicle," Journal of Guidance, Control, and Dynamics, vol. 30, no. 3, pp. 856-869, 2007.

[18] T. Williams, M. A. Bolender, D. B. Doman, and O. Morataya, "An aerothermal flexible mode analysis of a hypersonic vehicle," in Proceedings of the AIAA Atmospheric Flight Mechanics Conference and Exhibit, pp. 1391-1412, Keystone, Colo, USA, August 2006.

[19] A. Mannava and A. Serrani, "Further results on adaptive control design for non-minimum phase air-breathing hypersonic vehicles," in Proceedings of the American Control Conference, pp. 2255-2260, Seattle, Wash, USA, May 2017.

[20] Y. Zhang and B. Xian, "Continuous nonlinear asymptotic tracking control of an air-breathing hypersonic vehicle with flexible structural dynamics and external disturbances," Nonlinear Dynamics, vol. 83, no. 1-2, pp. 867-891, 2016.

[21] B. Tian, W. Fan, R. Su, and Q. Zong, "Nonlinear robust adaptive deterministic control for flexible hypersonic vehicles in the presence of input constraint," Asian Journal of Control, vol. 17, no. 6, pp. 2303-2316, 2015.

[22] Q. Zong, J. Wang, B. Tian, and Y. Tao, "Quasi-continuous highorder sliding mode controller and observer design for flexible hypersonic vehicle," Aerospace Science and Technology, vol. 27, no. 1, pp. 127-137, 2013.

[23] L. Fiorentini, A. Serrani, M. A. Bolender, and D. B. Doman, "Nonlinear robust adaptive control of flexible air-breathing hypersonic vehicles," Journal of Guidance, Control, and Dynamics, vol. 32, no. 2, pp. 401-416, 2009.

[24] H. Li, P. Lin, and D. Xu, "Control-oriented modeling for airbreathing hypersonic vehicle using parameterized configuration approach," Chinese Journal of Aeronautics, vol. 24, no. 1, pp. 81-89, 2011.

[25] X.-B. Zhang and Q. Zong, "Modeling and analysis of an airbreathing flexible hypersonic vehicle," Mathematical Problems in Engineering, vol. 2014, Article ID 264247, 9 pages, 2014.

[26] L. Fiorentini, A. Serrani, M. A. Bolender, and D. B. Doman, "Robust nonlinear sequential loop closure control design for an air-breathing hypersonic vehicle model," in Proceedings of the American Control Conference (ACC '08), pp. 3458-3463, IEEE, Seattle, Wash, USA, June 2008.

[27] K. P. Groves, A. Serrani, S. Yurkovich, M. A. Bolender, and D. B. Doman, "Anti-windup control for an air-breathing hypersonic vehicle model," AIAA Paper 2006-6557, 2006.

[28] B. Salimbahrami and B. Lohmann, "Krylov subspace methods in linear model order reduction: introduction and invariance properties," Scientific Report, 2002.

[29] C. Lanczos, "An iteration method for the solution of the eigenvalue problem of linear differential and integral operators," Journal of Research of the National Bureau of Standards, vol. 45, pp. 255-282, 1950.

[30] Z. Bai, "Krylov subspace techniques for reduced-order modeling of large-scale dynamical systems," Applied Numerical Mathematics, vol. 43, no. 1-2, pp. 9-44, 2002.

[31] R. Craig, Structural Dynamics: An Introduction to Computer Methods, Wiley, 1981.
[32] L. Fiorentini, Nonlinear adaptive controller design for airbreathing hypersonic vehicles [Degree Doctor of Philosophy thesis], Graduate School of the Ohio State University, Columbus, Ohio, USA, 2010.

[33] M. A. Bolender and D. B. Doman, "Flight path angle dynamics of air-breathing hypersonic vehicles," in Proceedings of the AIAA Guidance, Navigation, and Control Conference and Exhibit, AIAA Paper 2006-6692, pp. 4637-4661, August 2006.

[34] J. P. Hespanha, Linear Systems Theory, Princeton University Press, Princeton, NJ, USA, 2009.

[35] M. Oppenheimer, T. Skujins, M. Bolender, and D. Doman, "A flexible hypersonic vehicle model developed with piston theory," in Proceedings of the AIAA Atmospheric Flight Mechanics Conference and Exhibit, Hilton Head, SC, USA, August 2007.

[36] O. U. Rehman, I. R. Petersen, and B. Fidan, "Robust nonlinear control design of a hypersonic flight vehicle using minimax linear quadratic gaussian control," in Proceedings of the 49th IEEE Conference on Decision and Control (CDC '10), pp. 62196224, IEEE, Atlanta, Ga, USA, December 2010.

[37] L. Arnold, Stochastic Differential Equations: Theory and Applications, Wiley, 1973.

[38] W. Xiao, W. Zhang, and W. Xu, "Parameter estimation for fractional Ornstein-Uhlenbeck processes at discrete observation," Applied Mathematical Modelling, vol. 35, no. 9, pp. 4196-4207, 2011.

[39] M. Grigoriu and R. V. Field Jr., "A method for analysis of linear dynamic systems driven by stationary non-Gaussian noise with applications to turbulence-induced random vibration," Applied Mathematical Modelling, vol. 38, no. 1, pp. 336-354, 2014.

[40] A. D. Polyanin and V. F. Zaitsev, Handbook of Exact Solutions for Ordinary Differential Equations, Chapman \& Hall, 2002.

[41] Y. B. Shtessel, I. A. Shkolnikov, and A. Levant, "Smooth secondorder sliding modes: missile guidance application," Automatica, vol. 43, no. 8, pp. 1470-1476, 2007. 


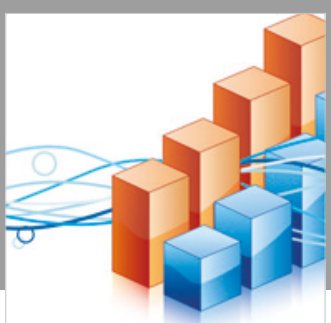

Advances in

Operations Research

\section{-n-m}
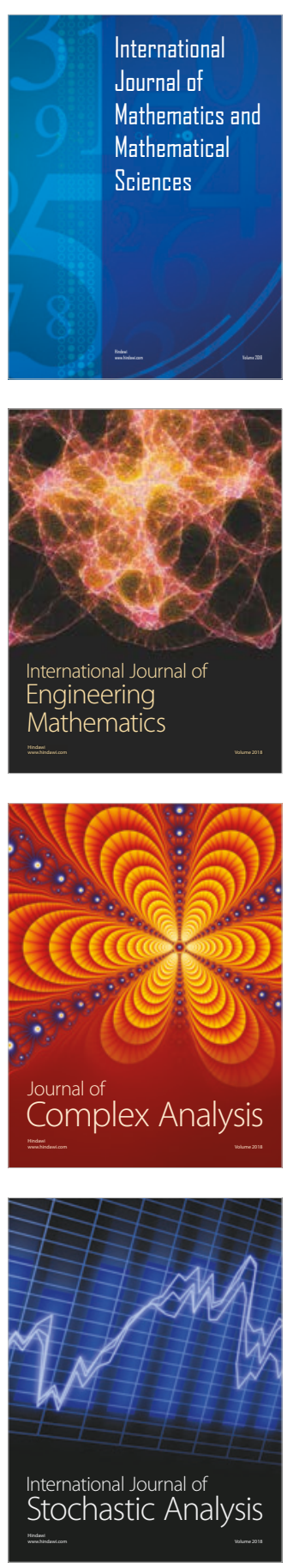
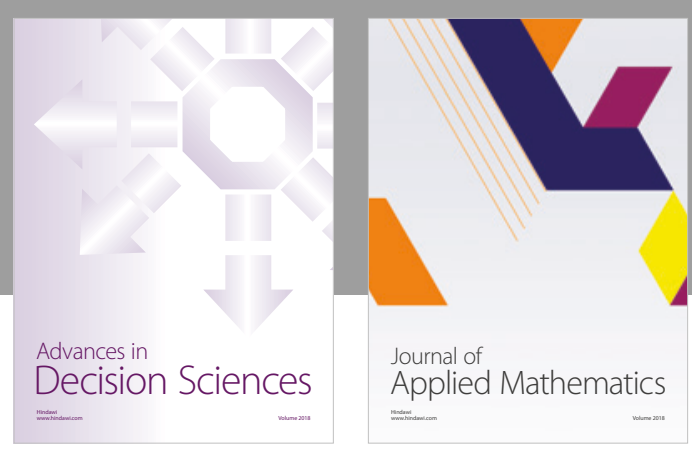

Journal of

Applied Mathematics
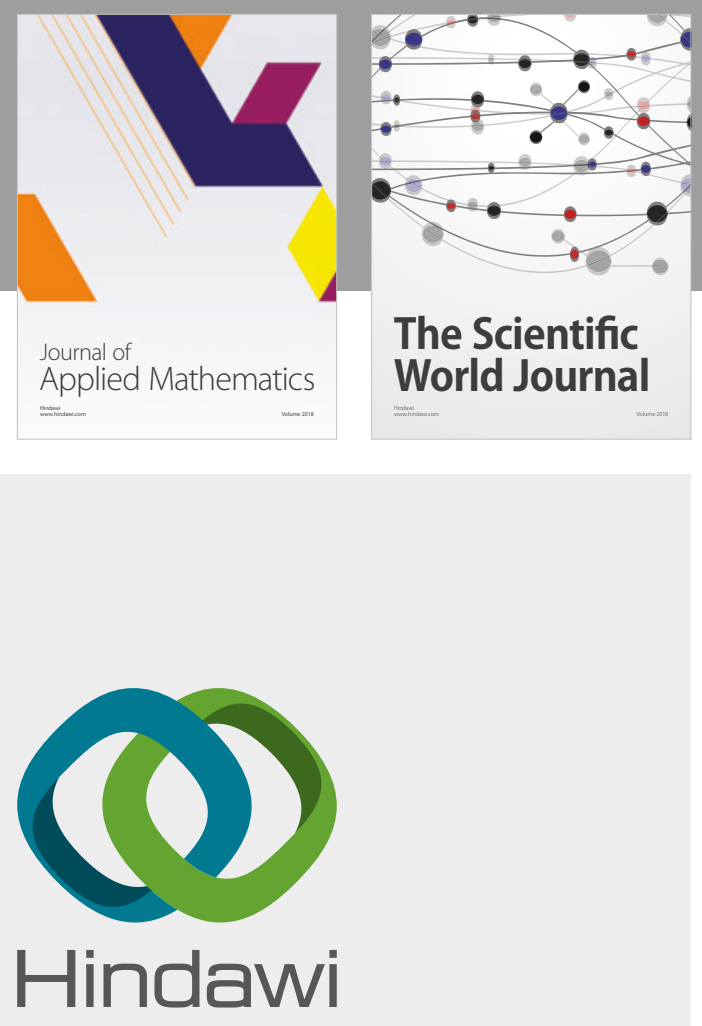

Submit your manuscripts at

www.hindawi.com

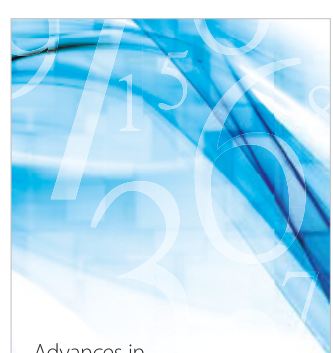

Advances in
Numerical Analysis
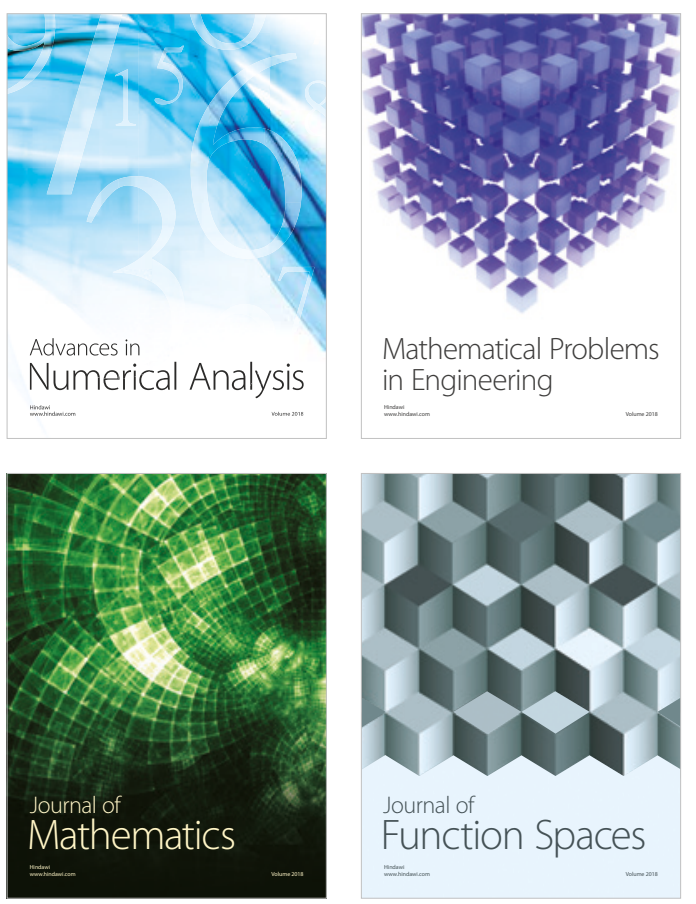

Mathematical Problems in Engineering

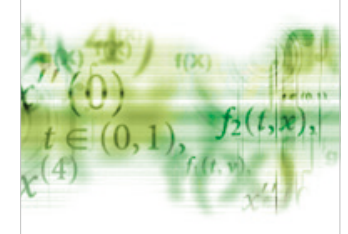

International Journal of

Differential Equations

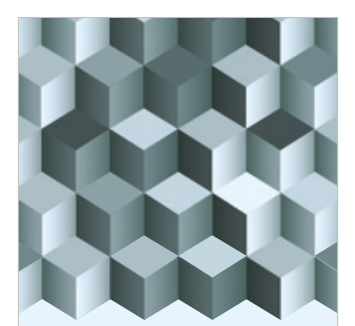

Journal of

Function Spaces

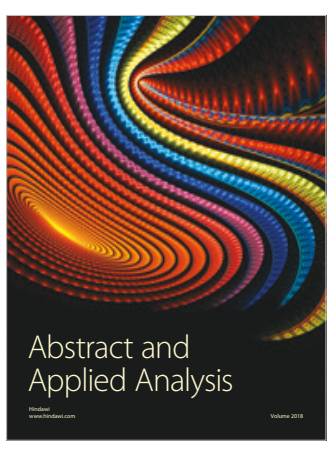

The Scientific

World Journal

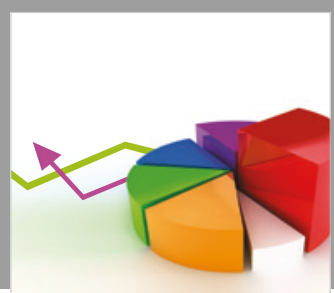

Journal of

Probability and Statistics
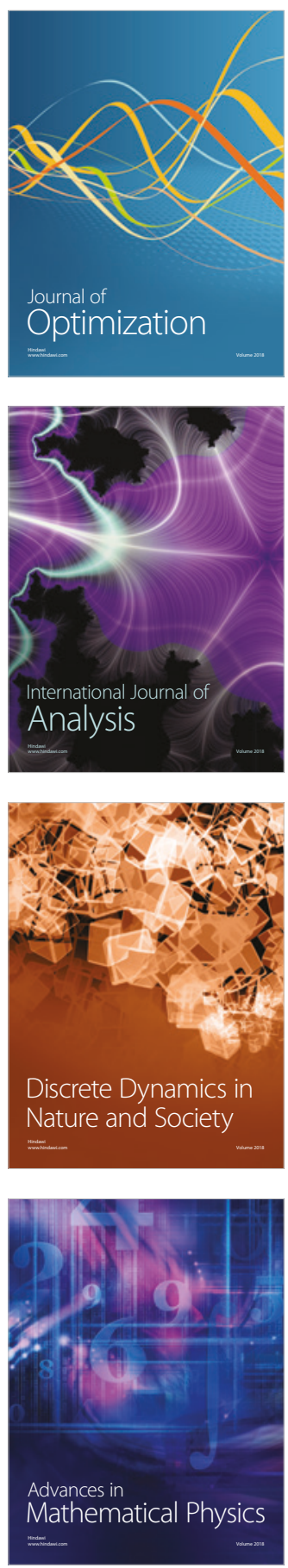